\title{
Prognostic value of serum uric acid and tumor response to induction chemotherapy in locally advanced nasopharyngeal carcinoma
}

Yuanji Xu ${ }^{1 \dagger}$, Zijie $\mathrm{Wu}^{1 \dagger}$, Wangzhong Ye ${ }^{1 \dagger}$, Youping Xiao ${ }^{2}$, Wei Zheng ${ }^{1}$, Qinyan Chen ${ }^{1}$, Penggang Bai ${ }^{1}$, Zhizhong Lin ${ }^{1}$ and Chuanben Chen ${ }^{1 *}$

\begin{abstract}
Background: To explore the combined predictive value of serum uric acid (SUA) and tumor response to induction chemotherapy (IC) in locally advanced nasopharyngeal carcinoma (LANPC) patients receiving IC followed by concurrent chemoradiation therapy (CCRT).

Methods: A total of 341 LANPC patients treated with IC + CCRT were enrolled in this retrospective study. Overall survival (OS), progression-free survival (PFS), locoregional relapse-free survival (LRFS), and distant metastasis-free survival (DMFS) were compared by the Kaplan-Meier analysis and the log-rank test, and multivariable survival analysis was carried out to investigate the independent prognostic factors.

Results: Univariate analysis showed that a low SUA level and unsatisfactory tumor response to two cycles of IC both were negative predictors for OS, PFS, and DMFS in patients with LANPC. multivariable analysis demonstrated that the SUA level after two cycles of IC was an independent prognostic factor for OS $(P=0.012)$ but of borderline significance for PFS and DMFS ( $P=0.055$ and $P=0.067$, respectively). Furthermore, tumor response to IC was of independent significance for predicting OS, PFS, and DMFS, respectively. Finally, LANPC patients with satisfactory tumor response and a high SUA level after two cycles of IC had a better OS, PFS, and DMFS than those with unsatisfactory tumor response and a low SUA level.

Conclusion: The SUA level and the tumor response to two cycles of IC had predictive value for LANPC patients treated with IC plus CCRT. However, more aggressive therapeutic strategies are recommended for those with a low SUA level and unsatisfactory tumor response to two cycles of IC.
\end{abstract}

Keywords: Nasopharyngeal carcinoma, Serum uric acid, Tumor response, Prognosis

\footnotetext{
* Correspondence: chenchuanben2010@126.com

${ }^{\dagger}$ Yuanji Xu, Zijie Wu and Wangzhong Ye contributed equally to this work. 'Department of Radiation Oncology, Fujian Medical University Cancer Hospital, Fujian Cancer Hospital, No.420, Fuma Road, Fuzhou, Fujian 350014, People's Republic of China

Full list of author information is available at the end of the article
}

(c) The Author(s). 2021 Open Access This article is licensed under a Creative Commons Attribution 4.0 International License, which permits use, sharing, adaptation, distribution and reproduction in any medium or format, as long as you give appropriate credit to the original author(s) and the source, provide a link to the Creative Commons licence, and indicate if changes were made. The images or other third party material in this article are included in the article's Creative Commons licence, unless indicated otherwise in a credit line to the material. If material is not included in the article's Creative Commons licence and your intended use is not permitted by statutory regulation or exceeds the permitted use, you will need to obtain permission directly from the copyright holder. To view a copy of this licence, visit http://creativecommons.org/licenses/by/4.0/. The Creative Commons Public Domain Dedication waiver (http://creativecommons.org/publicdomain/zero/1.0/) applies to the data made available in this article, unless otherwise stated in a credit line to the data. 


\section{Background}

Nasopharyngeal carcinoma (NPC) is a distinct malignant tumor with the highest number of incidences reported in South China. It is diverse from other types of head and neck squamous cell carcinoma in regard to epidemiology, biological characteristics, and clinical treatment [1]. The concurrent chemoradiotherapy (CCRT) followed by adjuvant chemotherapy or induction chemotherapy (IC) in addition to CCRT for locally advanced nasopharyngeal carcinoma (LANPC) was proposed as level 2A evidence by the National Comprehensive Cancer Network (NCCN) guidelines [2]. The increasing number of randomized controlled trials has demonstrated that the addition of IC to CCRT is of great importance in the treatment of LANPC patients in the intensity-modulated radiation therapy (IMRT) era, which reduces distant metastasis and subsequently improves the overall survival (OS) [3-5]. Nevertheless, 20\% 30\% patients with NPC would develop locoregional or distant metastasis, and distant metastasis was the most commonly seen failure pattern after treatment [6]. Therefore, explore novel prognostic factors to guide the clinical decision-making for a favorable and precise treatment after IC is an urgent requirement.

To date, the investigation on the prognostic factors of LANPC patients receiving IC remains largely unknown. In 2015, it was reported that measurable EBV-DNA loads as well as unfavorable tumor response (stable disease or disease progression) to IC were validated as negative predictors for LANPC patients [7]. In 2016, the tumor response to IC was subsequently determined as an independent prognostic factor for LANPC patients with IMRT [8]. In 2018, neutropenia during the first cycle of IC was found to be predictive for the poor survival of LANPC patients [9]. Recently, plasma EBV DNA load at IC completion was verified to be a robust and earlier survival outcome predictor for LANPC patients [10]. These observations confirmed that both plasma EBV-DNA load and the tumor response to IC had predictive value in LANPC patients that may be applied to direct the risk stratification and early treatment modification. Hence, in addition to plasma EBV DNA load, it is necessary to explore other biochemical indicators and predict the survival of patients with LANPC along with tumor response.

Uric acid is the final product of purine metabolism, which acts as a main antioxidant in serum and plays a vital role in defending cells from free radical-induced damage [11]. A previous study suggested that elevated uric acid levels might be attributed to increased purine metabolism by the action of xanthine oxidase, produced as a result of RNA-DNA breakdown in patients receiving radiotherapy [12]. In addition, the consumption of early appearing neutrophils in trauma or tumor lysis syndrome caused by radiochemotherapy could also elevate the serum uric acid (SUA) levels [13]. Moreover, aberrant SUA levels were involved with survival outcome in NPC patients in the IMRT era. Reportedly, the post-treatment SUA level was highly predictive of outcome in NPC patients treated by IMRT [14]. The pretreatment SUA level was an important biomarker in predicting distant metastasis in LANPC patients receiving IMRT [15]. Nevertheless, the predictive value of the SUA level after IC or the SUA levels associated with the tumor response to IC in LANPC patients undergoing IMRT has not yet been determined.

The present study aims to confirm whether the level of SUA after IC has prognostic significance in patients with LANPC. Furthermore, the level of SUA combined with tumor response to IC was investigated to evaluate the combined predictive value and guide the risk stratification for the decision of proper therapeutic schemes in LANPC.

\section{Materials and methods \\ Patients and pretreatment evaluation}

All 341 pathologically diagnosed NPC patients free of distant metastasis were enrolled in this retrospective study that was approved by Fujian Medical University Cancer Hospital institutional review board with a waiver of informed consent. All patients were treated with IC plus CCRT at our hospital from September 2014 to May 2017. The entry criteria were as follows: 1 ) records of the SUA levels of post-induction chemotherapy; 2) underwent a second MRI after two cycles of IC; 3) complete clinical data; 4) eliminate hyperuricemia or gout before treatment; 5) IC plus CCRT as the definitive treatment. The clinical information of all patients was provided in Table 1.

The routine pretreatment evaluation covered blood biochemistry, fiber nasopharyngoscopy, MRI of the nasopharynx and cervical region, chest CT scan, abdominal ultrasound, and whole-body bone scanning of the patients. Every patient was re-staged under the eighth edition of the International Union Against Cancer/ American Joint Committee on Cancer (UICC/AJCC) guidelines [16].

\section{Treatment}

A total of 341 patients were treated with the definitive IMRT. Details of IMRT in our institution have been described previously [17]. All patients were given IC followed by CCRT. The IC regimen included $2-4$ cycles of a platinum-based regimen with 2 or 3 chemotherapeutic drugs every 3 weeks. The recent chemotherapy regimen made up of a single platinum-based drug administered per 3 weeks. Moreover, 112 patients underwent adjuvant chemotherapy, and 43 patients received targeted therapy. Adjuvant chemotherapy and targeted 
Table 1 Clinical characteristics of 341 patients

\begin{tabular}{|c|c|c|c|c|}
\hline Characteristics & $\begin{array}{l}\text { SUA after two cycles of } \\
\text { IC }>327 \mu \mathrm{mol} / \mathrm{L}(\%)\end{array}$ & $\begin{array}{l}\text { SUA after two cycles of } \\
\text { IC } \leq 327 \mu \mathrm{mol} / \mathrm{L}(\%)\end{array}$ & PR/CR (\%) & SD/PD (\%) \\
\hline Total & $167(49.0 \%)$ & $174(51.0 \%)$ & $236(69.2 \%)$ & $105(30.8 \%)$ \\
\hline \multicolumn{5}{|l|}{ Gender } \\
\hline Female & $130(38.1 \%)$ & $101(29.6 \%)$ & $155(45.5 \%)$ & 76 (22.3\%) \\
\hline Male & 37 (10.9\%) & $73(21.4 \%)$ & 81 (23.7\%) & $29(8.5 \%)$ \\
\hline \multicolumn{5}{|l|}{ Age (years) } \\
\hline$\leq 50$ & $92(27.0 \%)$ & 97 (28.4\%) & $130(38.1 \%)$ & $59(17.3 \%)$ \\
\hline$>50$ & 75 (22.0\%) & 77 (22.6\%) & $106(31.1 \%)$ & 46 (13.5\%) \\
\hline \multicolumn{5}{|l|}{ Pathological type } \\
\hline WHO I & $0(0)$ & $2(0.6 \%)$ & $0(0)$ & $2(0.6 \%)$ \\
\hline WHO ॥ & $9(2.6 \%)$ & $9(2.6 \%)$ & $12(3.5 \%)$ & $6(1.8 \%)$ \\
\hline WHO III & $158(46.3 \%)$ & $163(47.8 \%)$ & $224(65.7 \%)$ & 97 (28.4\%) \\
\hline \multicolumn{5}{|l|}{ T stage } \\
\hline T1 & $24(7.0 \%)$ & $17(5.0 \%)$ & $28(8.2 \%)$ & $13(3.8 \%)$ \\
\hline $\mathrm{T} 2$ & $32(9.4 \%)$ & $38(11.1 \%)$ & $52(15.2 \%)$ & $18(5.3 \%)$ \\
\hline T3 & 65 (19.1\%) & $72(21.1 \%)$ & 99 (29.0\%) & $38(11.1 \%)$ \\
\hline T4 & $46(13.5 \%)$ & 47 (13.8\%) & 57 (16.7\%) & $36(10.6 \%)$ \\
\hline \multicolumn{5}{|l|}{ N stage } \\
\hline NO & $11(3.2 \%)$ & $7(2.1 \%)$ & $11(3.2 \%)$ & $7(2.1 \%)$ \\
\hline N1 & 49 (14.4\%) & 68 (19.9\%) & $80(23.5 \%)$ & 37 (10.9\%) \\
\hline N2 & 87 (25.5\%) & 79 (23.2\%) & $115(33.7 \%)$ & $51(15.0 \%)$ \\
\hline N3 & $20(5.9 \%)$ & $20(5.9 \%)$ & $30(8.8 \%)$ & $10(2.9 \%)$ \\
\hline \multicolumn{5}{|c|}{ Chemotherapy cycles } \\
\hline$\leq 3$ & $31(9.1 \%)$ & $31(9.1 \%)$ & $41(12.0 \%)$ & $21(6.2 \%)$ \\
\hline$>3$ & $136(39.9 \%)$ & $143(41.9 \%)$ & 195 (57.2\%) & 84 (24.6\%) \\
\hline
\end{tabular}

Abbreviations: $C R$ complete response, $I C$ induction chemotherapy, $P D$ disease progression, $P R$ partial response, $S D$ stable disease, $S U A$ serum uric acid

therapy were chosen less often because of poor compliance and high cost to patients with NPC.

\section{Measurement of the SUA level and tumor response assessment}

The SUA level was measured after two cycles of IC using an enzyme kinetics kit (Roche Diagnostics, Germany) by a Modular PP model automated analyzer. Each patient underwent MRI of the nasopharynx as well as cervical region at the pre- and post-induction chemotherapy with two cycles, respectively. Subsequently, the tumor response was assessed by two independent radiologists in a double-blinded manner based on the Response Evaluation Criteria in Solid Tumors criteria 1.1 (RECIST 1.1) [18]. Any tumor response with conflicting results was resolved by consensus. Satisfactory tumor response was determined as complete response (CR) and partial response (PR), whereas unsatisfactory tumor response was classified as stable disease (SD) and disease progression (PD).

\section{Follow-up and statistical analysis}

The follow-up duration was measured from day 1 of the diagnosis of NPC until death or the last follow-up of the patient. The survival endpoints included Overall survival (OS), progression-free survival (PFS), distant metastasisfree survival (DMFS), and locoregional relapse-free survival (LRFS). Beginning from day 1 of treatment, OS was defined as the time to the date of death or patient censoring, whichever occurred first; PFS, to disease failure, death from any cause, or patient censoring, whichever occurred first; DMFS, to distant failure, death from any cause, or patient censoring, whichever occurred first; and LRFS, to local failure or regional failure, death from any cause or patient censoring, whichever occurred first. Patients were evaluated once every 3 months within the first 3 years of follow-up and every 6 months thereafter until death. The median follow-up time was 41 months (range, 3.0-62.0 months).

The SPSS 26.0 software package (SPSS Inc., Chicago, IL, USA) was used for all statistical analyses. Survival analyses were performed by the Kaplan-Meier method, 
and the log-rank test was employed to make a comparison between the two groups. A Cox proportional hazard model was carried out for the multivariable analyses of the following variables: gender (male versus female), age ( $\leq 50$ years versus $>50$ years), pathological type (WHO III vs WHO III), T stage (T1-T2 vs T3-T4), N stage (N01 vs $\mathrm{N} 2-3)$, chemotherapy cycle ( $\leq 3$ versus $>3$ ), the SUA level $(\leq 327 \mu \mathrm{mol} / \mathrm{L}$ versus $>327 \mu \mathrm{mol} / \mathrm{L})$, and tumor response to IC (CR/PR versus $\mathrm{SD} / \mathrm{PD})$. All statistical tests were 2 -sided, and a $P$-value less than 0.05 was confirmed to be statistically significant.

\section{Results}

\section{Patient characteristics and outcome}

Among the 341 patients, the male $(n=231)$-to-female $(n=110)$ ratio was 2.1:1. The median age of the cohort was 48 (range: $10-78$ ) years. Histologically, $94.1 \%$ of the patients had World Health Organization (WHO) type III disease, $5.3 \%$ had WHO type II disease, and $0.6 \%$ with WHO I type disease. Other clinical characteristics, including $\mathrm{T}$ stage, $\mathrm{N}$ stage, clinical stage, and chemotherapy cycles, were provided in Table 1.

The median follow-up duration was 41 (range: 3-62) months. By the end of follow-up, 19 (5.6\%) patients experienced local or regional recurrence and 49 (14.4\%) developed distant metastasis, including 16 cases of pulmonary metastasis, 10 cases of bone metastasis, 14 cases of liver metastasis, 8 cases of multiregional metastasis, and 1 case of metastasis at another site. Finally, 42 (12.3\%) patients deceased, including 41 with tumor progression and 1 by car accident.

\section{Correlation between SUA level after IC and clinical outcome}

The median SUA level after two cycles of IC for all patients was 327 (range: 144-585) $\mu \mathrm{mol} / \mathrm{L}$. The 3-year OS, PFS, and DMFS of patients with the SUA level > $327 \mu \mathrm{mol} / \mathrm{L}$ were significantly higher than those with the SUA level $\leq 327 \mu \mathrm{mol} / \mathrm{L}$ after two cycles of IC: 3-year OS, 95.8\% (95\% confidence interval (CI): 57.4-60.6\%) versus $88.4 \%$ (95\% CI: $52.1-56.5 \%, P=0.006$ ) (Fig. 1a);
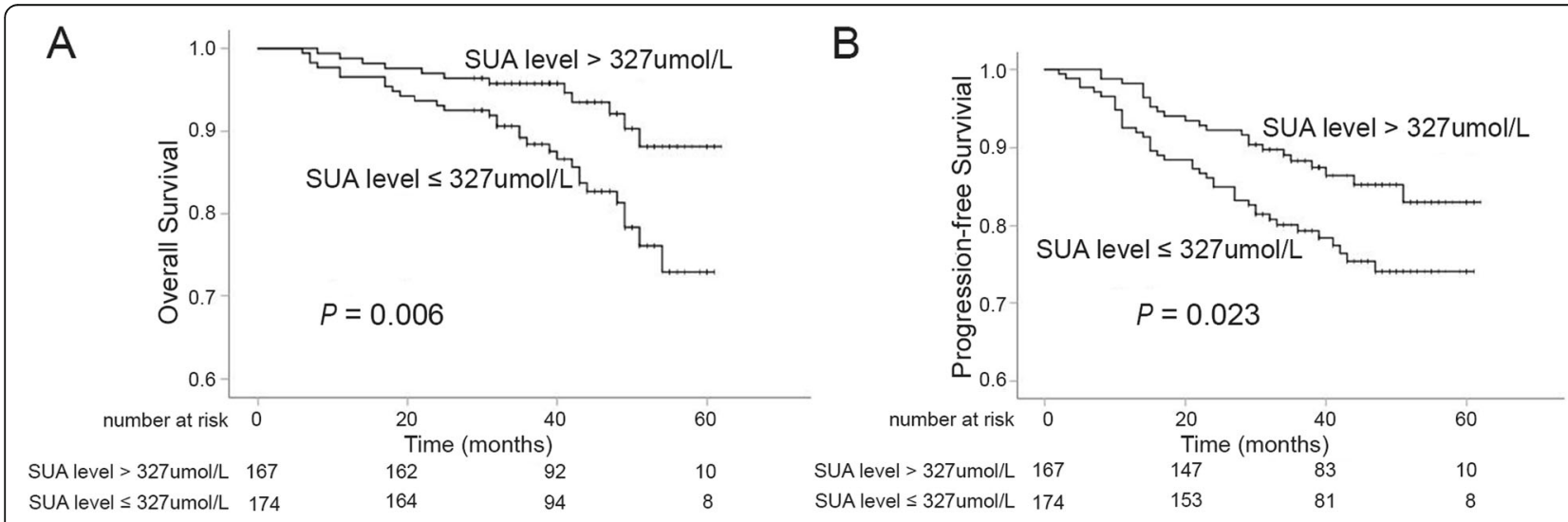

C

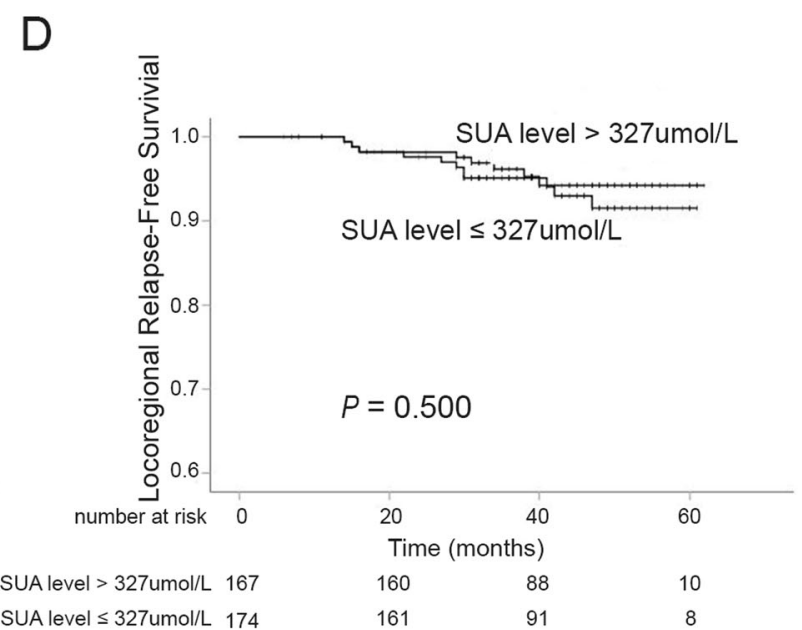

SUA level > 327umol/L 167
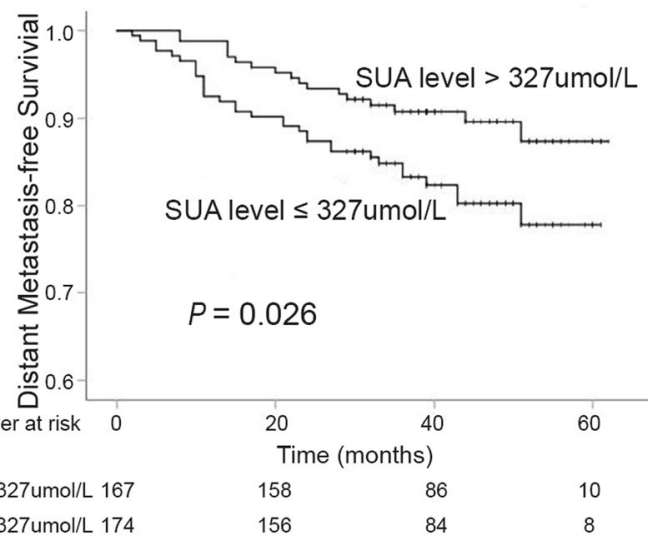

$P=0.026$

Fig. 1 The contrast of OS (a), PFS (b), DMFS (c), and LRFS (d) between LANPC patients with high and low SUA level after IC 
3-year PFS, 88.3\% (95\% CI: 54.1-58.5\%) versus 79.3\% (95\% CI: 48.4-53.9\%, $P=0.023$ ) (Fig. 1b); 3-year DMFS, 90.8\% (95\% CI: 55.7-59.7\%) versus 83.2\% (95\% CI: 50.3$55.5 \%, P=0.026$ ) (Fig. 1c). However, the 3-year LRFS was not significantly different between the above two groups: 96.2\% (95\% CI: 58.8-61.4\%) versus 95.1\% (95\% CI: $57.1-59.9 \%, P=0.500$ ) (Fig. $1 \mathrm{~d}$ ).

\section{Correlation between tumor response to IC and clinical outcome}

Patients with unsatisfied tumor response had an unfavorable 3-year OS, PFS, and DMFS as a comparison to those with satisfied tumor response: 3-year OS, 94.1\% (95\% CI: 55.9-58.9\%) versus $87.3 \%$ (95\% CI: $51.3-57.4 \%, P=0.019$ ) (Fig. 2a); 3-year PFS, 87.1\% (95\% CI: 53.6-57.3\%) versus 76.0\% (95\% CI: 45.5-53.1\%, $P<0.001$ ) (Fig. 2b); 3-year DMFS, 90.7\% (95\% CI: 55.2-58.6\%) versus 78.6\% (95\% CI: 47.6-54.8\%, $P<0.001$ ) (Fig. 2c). Nevertheless, the 3 -year LRFS was not markedly different between the above two groups: $96.0 \%$ (95\% CI: 58.0-60.2\%) versus 94.7\% (95\% CI: 57.1-61.0\%, $P=0.314$ ) (Fig. 2d).
Analysis of the independent predictive factors for LANPC patients after two cycles of IC

To investigate the independent prognostic factors for LANPC patients after two cycles of IC, multivariable analysis was conducted. Consequently, the SUA level after two cycles of IC, tumor response to IC, $\mathrm{N}$ stage, and age were found to be independent prognostic factors for $\mathrm{OS}(P=0.012, P=0.036, P=0.005$ and $P=0.025$, respectively) (Table 2). In addition, tumor response to IC was only observed to be an independent predictive factor for PFS $(P=0.001)$ (Table 2). Furthermore, both the tumor response to IC and N stage were identified as independent prognostic factors for DMFS $(P=0.001$ and $P=0.011$, respectively) (Table 2 ). Notably, the level of SUA was of borderline significance for PFS and DMFS ( $P=0.055$ and $P=0.067$, respectively) (Table 2 ).

Correlation between combined plasma uric acid level and tumor response and clinical outcome

Based on the level of SUA and tumor response, patients were divided into three subgroups: (1) high
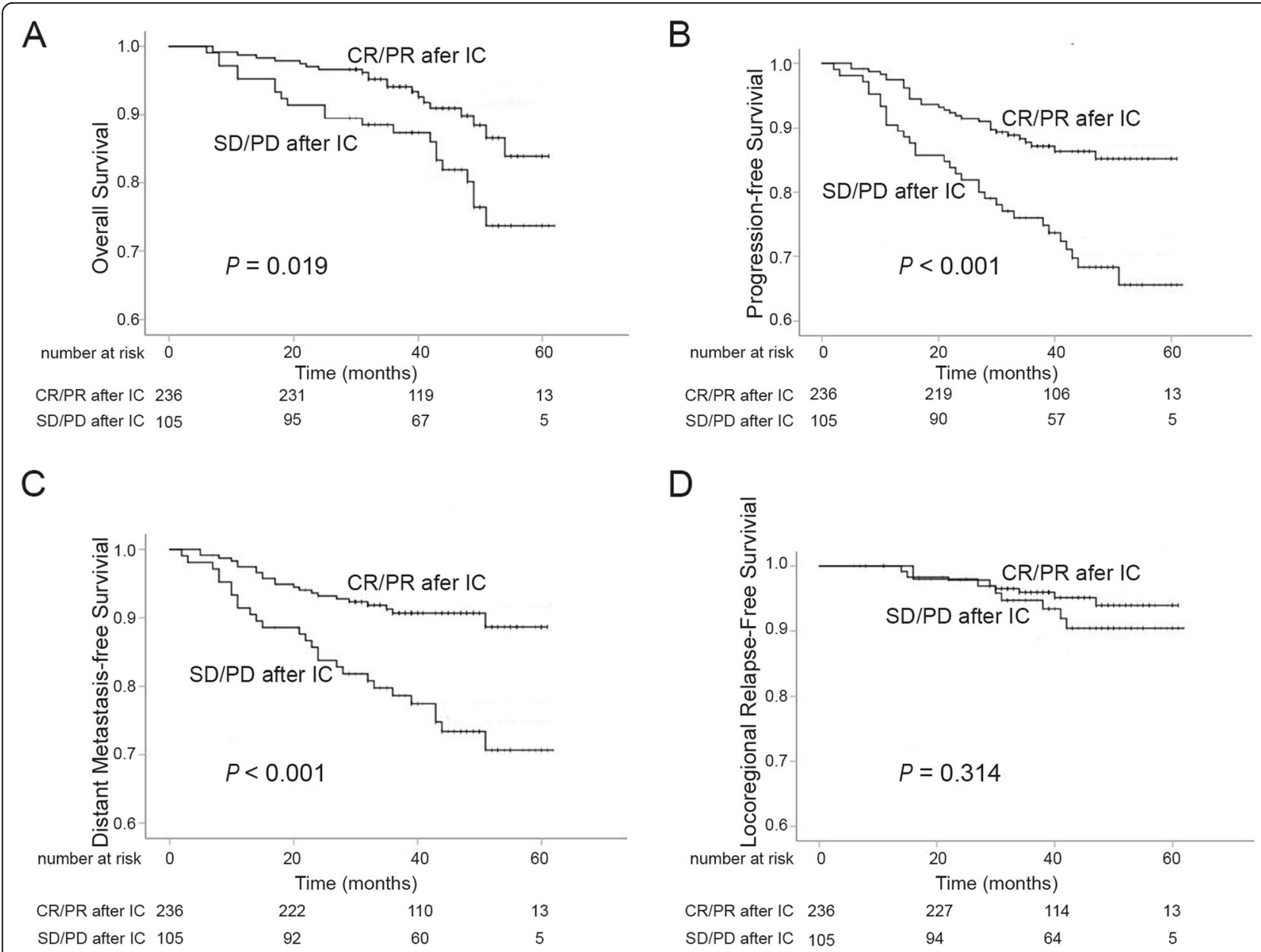

Fig. 2 The contrast of OS (a), PFS (b), DMFS (c), and LRFS (d) between LANPC patients with a satisfactory and unsatisfactory response to IC 
Table 2 Multivariate analyses of independent significance of experimental intervention on clinical outcome

\begin{tabular}{|c|c|c|c|c|c|c|}
\hline \multirow[t]{2}{*}{ Variables } & \multicolumn{2}{|l|}{ OS(42/341) } & \multicolumn{2}{|l|}{ PFS(63/341) } & \multicolumn{2}{|l|}{ DMFS(49/341) } \\
\hline & HR $(95 \% \mathrm{Cl})$ & $P$ & HR $(95 \% \mathrm{Cl})$ & $P$ & $\mathrm{HR}(95 \% \mathrm{Cl})$ & $P$ \\
\hline Gender (male vs female) & $1.053(0.546-2.031)$ & 0.877 & $0.904(0.518-1.578)$ & 0.723 & $0.737(0.384-1.412)$ & 0.357 \\
\hline Age $(\leq 50$ vs $>50)$ & $2.039(1.092-3.807)$ & 0.025 & $1.512(0.918-2.492)$ & 0.105 & $1.342(0.761-2.367)$ & 0.310 \\
\hline Pathological type (WHO I-II vs WHO III) & $2.295(0.361-14.583)$ & 0.379 & $1.961(0.531-7.246)$ & 0.313 & $1.532(0.420-5.588)$ & 0.518 \\
\hline T stage (T1-T2 vs T3-T4) & $1.329(0.944-1.870)$ & 0.103 & $1.123(0.866-1.457)$ & 0.382 & $1.129(0.838-1.520)$ & 0.425 \\
\hline N stage (N0-1 vs N2-3) & $1.766(1.186-2.630)$ & 0.005 & $1.378(0.990-1.920)$ & 0.058 & $1.634(1.117-2.391)$ & 0.011 \\
\hline Chemotherapy cycle ( $\leq 3$ vs $>3$ ) & $2.381(0.910-6.226)$ & 0.077 & $1.747(0.830-3.676)$ & 0.142 & $1.808(0.767-4.264)$ & 0.176 \\
\hline SUA level ( $\leq 327$ vs $>327)$ & $2.385(1.208-4.707)$ & 0.012 & $1.658(0.989-2.779)$ & 0.055 & $1.744(0.964-3.156)$ & 0.066 \\
\hline Tumor response to IC (CR/PR vs SD/PD) & $1.915(1.044-3.648)$ & 0.036 & $2.381(1.408-3.816)$ & 0.001 & $2.764(1.554-4.851)$ & 0.001 \\
\hline
\end{tabular}

Abbreviations: $C l$ confidence interval, $C R$ complete response, DMFS distant metastasis-free survival, $H R$ hazard ratio, IC induction chemotherapy, OS overall survival, $P D$ disease progression, $P F S$ progression-free survival, $P R$ partial response, $S D$ stable disease, SUA serum uric acid

SUA level $(>327 \mu \mathrm{mol} / \mathrm{L})$ and $\mathrm{CR} / \mathrm{PR}(n=123)$; (2) low SUA level $(\leq 327 \mu \mathrm{mol} / \mathrm{L})$ and $\mathrm{CR} / \mathrm{PR}$ or a high SUA level $(>327 \mu \mathrm{mol} / \mathrm{L})$ and $\mathrm{SD} / \mathrm{PD}(n=157) ;(3)$ low SUA level $(\leq 327 \mu \mathrm{mol} / \mathrm{L})$ and SD/PD $(n=61)$. The 3-year OS rates for the three subgroups were 97.6, 90.4, and $84.9 \%$, respectively $(P=0.002)$ (Fig. 3a). The 3-year PFS rates for the three subgroups were 90.6, 83.0, and $71.9 \%$, respectively $(P<0.001)$ (Fig. $3 \mathrm{~b})$. The 3-year DMFS rates for the three subgroups were $93.3,86.7$, and $74.9 \%$, respectively $(P<0.001)$ (Fig. 3c). However, the 3-year LRFS rates were similar among the three patient groups: 96.5, 95.3, and $94.4 \%$, respectively $(P=0.523)$ (Fig. $3 \mathrm{~d})$.

\section{Discussion}

At present, the addition of IC to the CCRT has been demonstrated as an attractive multidisciplinary approach for the treatment of LANPC [3-5]. Accordingly, few prognostic factors in LANPC patients treated with IC have been investigated. The tumor response to IC was shown to be an independent prognostic factor for LANP $C$ patients underwent IMRT $[7,8]$. In addition, the SUA level was confirmed to be elevated because of the radiochemotherapy that led to tumor trauma or lysis [13]. However, the prognostic value of SUA after IC or the SUA levels combined with the tumor response to IC in LANPC patients remains unclear. The current study represents the first report to explore the combined prognostic value of the SUA levels and the tumor response to IC in LANPC patients. In this study, it was shown that a high SUA level after two cycles of IC was involved in significantly improved OS, while PFS and DMFS in LANPC patients exhibited borderline significance. Furthermore, the satisfactory tumor response to IC was correlated with significantly improved OS, PFS, and DMFS in LANPC patients. Moreover, the combination of the SUA level with tumor response to IC represented an optimal predictor of OS, PFS, and DMFS, respectively, in patients with a high SUA level and satisfactory tumor response to IC. These results provided a clinical reference for further guiding the risk stratification and early treatment modification for LANPC.

In this study, both univariate and multivariable analyses revealed that the high SUA level after two cycles of IC $(>327 \mu \mathrm{mol} / \mathrm{L})$ was a positive prognostic factor for NPC as a comparison to the SUA level $\leq 327 \mu \mathrm{mol} / \mathrm{L}$ with a 2.385 -fold increased risk of death. Additionally, borderline significant differences were discovered between the high and low SUA levels with respect to the risk of disease failure and distant failure. However, the SUA level was not correlated with the risk of locoregional failure. Uric acid is considered as an antioxidant, which has the effects of eliminating reactive oxygen free radicals, protecting DNA damage, reducing the cell migration ability, and regulating tumor cell death $[11,19]$. Chemotherapy is always associated with increased damage to DNA and reduced tumor burden of the patients, which might finally elevate the SUA levels [20]. In addition, uric acid mediates the cytotoxicity of natural killer and $\mathrm{T}$ cells by inducing vital stress-inducing ligands' expression on cancer cells [21]. Thus, it can be hypothesized that uric acid may exert a preventive effect against the development of cancer. Hence, the SUA levels after chemotherapy reflect the efficacy of chemotherapy in patients with NPC, which leads us to propose that the level of SUA after chemotherapy might be closely related to the prognosis. The level of SUA at the pretreatment and the completion of the definitive IMRT has been reported to be closely tied up with the prognosis in NPC $[14,15]$. Nevertheless, additional studies are essential to elucidate the mechanisms associated with high SUA levels after IC and improved survival in LANP $C$ patients treated by IC plus CCRT.

According to the current findings, LANPC patients with SD/PD to IC presented an unfavorable OS, PFS, and DMFS than those with CR/PR to IC. Moreover, the tumor response to IC was identified to be an independent prognostic factor for OS, PFS, and DMFS, 


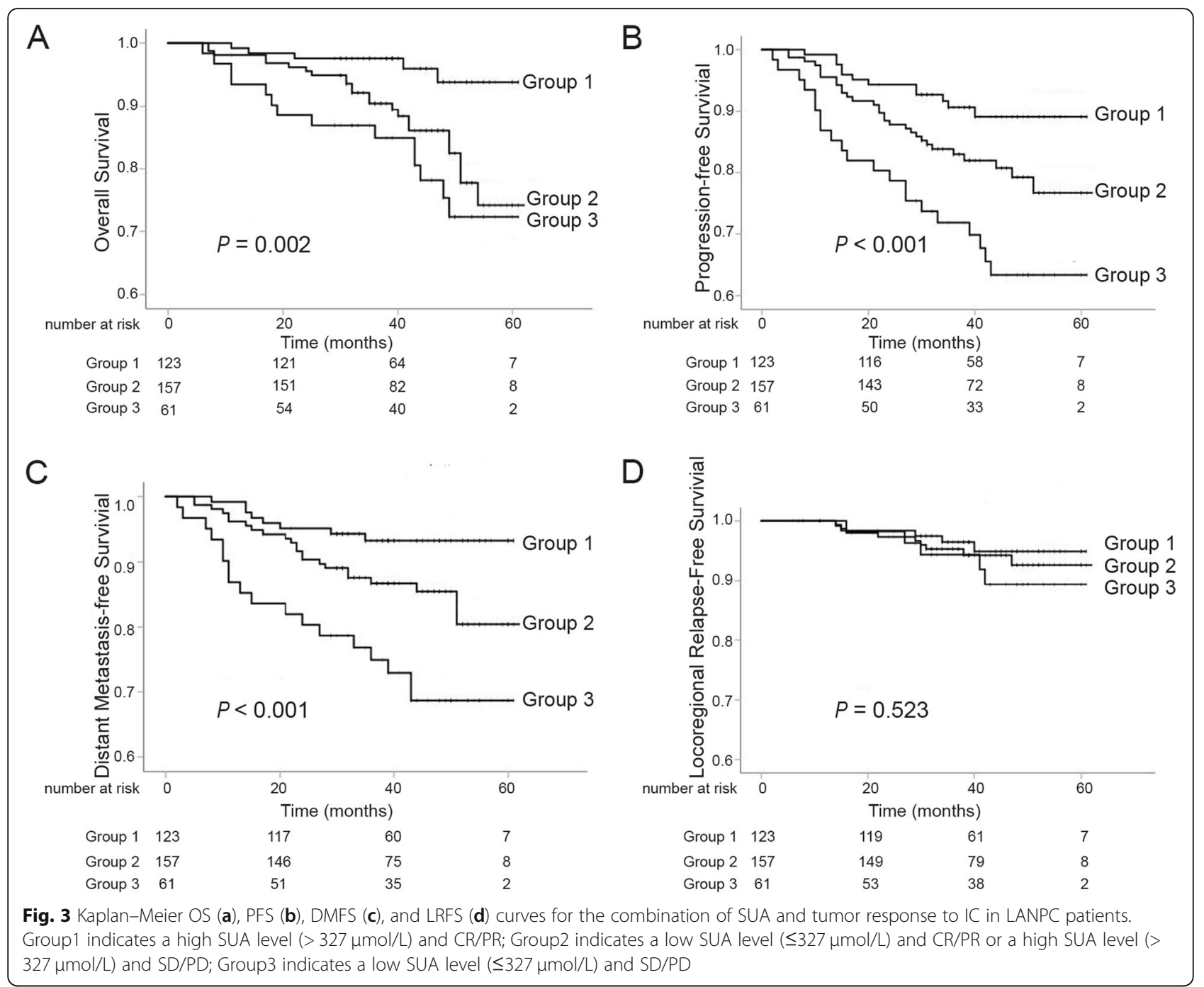

respectively. To our knowledge, the prognostic significance of tumor response to chemotherapy in various malignancies has been confirmed [22, 23]. Subsequently, the tumor response to IC was demonstrated to be an independent prognostic factor for LANPC patients with IMRT [7, 8], which was similar to our results. Intriguingly, the tumor response to chemotherapy basically represented the changes in gross tumor volume. Typically, patients with CR/PR to IC implied that the tumor volume shrank dramatically or disappeared completely. In contrast, patients with SD/PD to IC showed that the tumor volume shrank insignificantly or rather increased. Several studies demonstrated the prognostic value of changes in the tumor volume in different types of cancers $[24,25]$. Therefore, the prognostic value of tumor response to IC in LANPC patients can be elucidated. Taken together, it can be deduced that the SUA level and tumor response to IC were closely involved in IC, and hence, the combined predictive value of the SUA level and tumor response to IC needs deeper investigation.

To explore the prognostic value of combining SUA levels with tumor response to IC, LANPC patients were divided into three subgroups. The outcomes of recent study showed that the subgroup of patients with a high SUA level and CR/PR exhibited improved OS, PFS, and DMFS; this was defined as the low-risk group. The subgroup of patients with a low SUA level and SD/PD showed poor prognosis and was classified as the highrisk group. These phenomena demonstrated that the combination of SUA levels and tumor response to IC had significant prognostic value in LANPC, which might help in differentiating the risk stratification and improving the prognosis after IC. The high-risk group seemed insensitive to IC, needing more aggressive treatment strategies after IC: (1) an increased dose of RT, (2) the administration of an additional target agent during CCRT, like cetuximab that reported to be a feasible 
strategy against LANPC [26], (3) the inclusion of adjuvant chemotherapy, or (4) the addition of immunotherapy $[27,28]$. For the low-risk group, the radiation dose could be decreased to decrease the side effects of radiotherapy. Furthermore, it is feasible for the treating physicians to decide whether to continue or change the IC regimen based on the SUA level and tumor response after two cycles of IC, which could lower the expenses of the treatment and the complications of chemotherapy.

The current study showed that both the $\mathrm{N}$ stage and age were independent prognostic factors for OS by multivariable analyses. In addition, the $\mathrm{N}$ stage was also verified to be an independent prognostic factor for DMFS. At present, the $\mathrm{N}$ stage is the most crucial risk factor for death and distant metastasis [29]. Furthermore, elderly patients are more likely to develop disease failure and die [30], which is in agreement with the outcome of this study.

Nevertheless, our study has several limitations. First, the tumor response was assessed by the radiologists according to RECIST 1.1 [18]; however, it is hard to evaluate the tumor response in LANPC patients with skull base invasion. In this study, the abnormal MRI signal of the skull base was observed after soft tumor regression, which made it difficult to identify whether the skull base was actually infiltrated. Under such circumstances, the skull base was not included in the measurement of tumor length that may lead to an inaccurate evaluation of the tumor response. Second, the median follow-up duration was only 41 months; thus, it is of great importance to prolong the follow-up time to assess the longterm outcomes of patients with LANPC. Third, other biomedical biomarkers, including plasma EBV-DNA, ALP, and LDH, were not evaluated in our study. Therefore, developing a predictive nomogram model to investigate the array of prognostic factors is imperative.

\section{Conclusion}

In conclusion, the SUA level and tumor response to two cycles of IC have predictive value for LANPC patients receiving IC plus CCRT. However, more aggressive therapeutic strategies are recommended for LANPC patients with a low SUA level and unsatisfactory tumor response to two cycles of IC.

\section{Abbreviations}

NPC: Nasopharyngeal carcinoma; CCRT: Concurrent chemoradiotherapy; IC: Induction chemotherapy; LANPC: Locally advanced nasopharyngeal carcinoma; NCCN: National Comprehensive Cancer Network; IMRT: Intensitymodulated radiation therapy; OS: Overall survival; SUA: Serum uric acid; UICC/AJCC: International Union Against Cancer/American Joint Committee on Cancer; RECIST 1.1: Response Evaluation Criteria in Solid Tumors criteria 1.1; CR: Complete response; PR: Partial response; SD: Stable disease; PD: Disease progression; PFS: Progression-free survival; DMFS: Distant metastasis-free survival; LRFS: Locoregional relapse-free survival; $\mathrm{Cl}$ : Confidence interval
}

\section{Acknowledgements}

We are grateful to our patients and staff members who participated in the patient care to make this project available.

\section{Authors' contributions}

Yj X: design experiment, perform research, analyze data and make relevant statistics, draft the manuscript and make repeated modifications. ZW: participate in experimental design, analyze data and make relevant statistics, complete part of the manuscript revision work. WY: analyze data and make relevant statistics. Yp X: revised the manuscript several times, language polishing. ZL: acquisition parameters, participate in research. PB: acquisition parameters, make relevant statistics. QC: acquisition parameters, data measurement. WZ: acquisition parameters, data measurement. CC: design experiment, critically review the intellectual content of the manuscript, financial support for research, full guidance. All authors read and approved the final manuscript.

\section{Funding}

This project was funded by Joints Funds for the Innovation of Science and Technology, Fujian Province (Grant number: 2018Y9107, 2019Y9034), Natural Science Foundation of Fujian Province, China (Grant number: 2019 J01201, 2019 J05140), Fujian Provincial Health Technology Project (Grant number: 2018-CX-11, 2018-ZQN-19, 2019-ZQN-14), Startup for Scientific Research, Fujian Medical University (Grant number: 2018QH1222), and Science and Technology Program of Fujian Province, China (Grant number: 2018Y2003).

\section{Availability of data and materials}

The data that support the findings of this study are available from Fujian Medical University Cancer Hospital, but restrictions apply to the availability of these data, which were used under license for the current research, and so are not publicly available. Data are, however, available from the corresponding authors upon reasonable request and with permission of Fujian Medical University Cancer Hospital.

\section{Declarations}

\section{Ethics approval and consent to participate}

This study is a retrospective observational study and was conducted in accordance with the relevant guidelines, regulations, and the Declaration of Helsinki. The methods and procedures for this study were approved by Fujian Medical University Cancer Hospital institutional review board with a waiver of informed consent (Ref no. SQ2019-018-01).

\section{Consent for publication}

Not applicable.

\section{Competing interests}

The authors declare that they have no competing interests.

\section{Author details}

'Department of Radiation Oncology, Fujian Medical University Cancer Hospital, Fujian Cancer Hospital, No.420, Fuma Road, Fuzhou, Fujian 350014, People's Republic of China. ${ }^{2}$ Department of Radiology, Fujian Medical University Cancer Hospital, Fujian Cancer Hospital, Fuzhou, Fujian, People's Republic of China.

Received: 9 March 2021 Accepted: 29 April 2021

Published online: 08 May 2021

\section{References}

1. Chua MLK, Wee JTS, Hui EP, Chan ATC. Nasopharyngeal carcinoma. Lancet 2016;387(10022):1012-24. https://doi.org/10.1016/S0140-6736(15)00055-0.

2. Colevas AD, Yom SS, Pfister DG, Spencer S, Adelstein D, Adkins D, et al. NCCN guidelines insights: head and neck cancers, version 1.2018. J Natl Compr Cancer Netw. 2018;16(5):479-90. https://doi.org/10.6004/jnccn.201 8.0026.

3. Sun Y, Li WF, Chen NY, Zhang N, Hu GQ, Xie FY, et al. Induction chemotherapy plus concurrent chemoradiotherapy versus concurrent chemoradiotherapy alone in locoregionally advanced nasopharyngeal carcinoma: a phase 3, multicentre, randomised controlled trial. Lancet Oncol. 2016;17(11):1509-20. https://doi.org/10.1016/S1470-2045(16)30410-7. 
4. Zhang Y, Chen L, Hu GQ, Zhang N, Zhu XD, Yang KY, et al. Gemcitabine and Cisplatin induction chemotherapy in nasopharyngeal carcinoma. N Engl J Med. 2019;381(12):1124-35. https://doi.org/10.1056/NEJMoa1905287.

5. Chen YP, Chan ATC, Le QT, Blanchard P, Sun Y, Ma J. Nasopharyngeal carcinoma. Lancet. 2019;394(10192):64-80. https://doi.org/10.1016/S01406736(19)30956-0

6. Ribassin-Majed L, Marguet S, Lee AWM, Ng WT, Ma J, Chan ATC, et al. What is the best treatment of locally advanced nasopharyngeal carcinoma? An individual patient data network meta-analysis. J Clin Oncol. 2017;35(5):498505. https://doi.org/10.1200/JCO.2016.67.4119.

7. Liu LT, Tang LQ, Chen QY, Zhang L, Guo SS, Guo L, et al. The prognostic value of plasma Epstein-Barr viral DNA and tumor response to Neoadjuvant chemotherapy in advanced-stage nasopharyngeal carcinoma. Int J Radiat Oncol Biol Phys. 2015;93(4):862-9. https://doi. org/10.1016/j.jijobp.2015.08.003.

8. Peng $H$, Chen L, Zhang Y, Li WF, Mao YP, Liu X, et al. The tumour response to induction chemotherapy has prognostic value for long-term survival outcomes after intensity-modulated radiation therapy in nasopharyngeal carcinoma. Sci Rep. 2016;6(1):24835. https://doi.org/10.1038/srep24835.

9. Xu C, Yang SP, Zhang Y, Tang LL, Zhou GQ, Liu X, et al. Neutropenia during the first cycle of induction chemotherapy is prognostic for poor survival in Locoregionally advanced nasopharyngeal carcinoma: a real-world study in an endemic area. Cancer Res Treat. 2018;50(3):777-90. https://doi.org/1 $0.4143 /$ crt.2017.255.

10. Huang CL, Sun ZQ, Guo R, Liu X, Mao YP, Peng H, et al. Plasma Epstein-Barr virus DNA load after induction chemotherapy predicts outcome in Locoregionally advanced nasopharyngeal carcinoma. Int J Radiat Oncol Biol Phys. 2019;104(2):355-61. https://doi.org/10.1016/j.jirobp.2019.01.007.

11. Fini MA, Elias A, Johnson RJ, Wright RM. Contribution of uric acid to cancer risk, recurrence, and mortality. Clin Transl Med. 2012;1(1):16. https://doi.org/1 0.1186/2001-1326-1-16.

12. Crohns M, Saarelainen S, Kankaanranta H, Moilanen E, Alho H, KellokumpuLehtinen P. Local and systemic oxidant/antioxidant status before and during lung cancer radiotherapy. Free Radic Res. 2009;43(7):646-57. https:// doi.org/10.1080/10715760902942824.

13. Haen SP, Eyb V, Mirza N, Naumann A, Peter A, Löffler MW, et al. Uric acid as a novel biomarker for bone-marrow function and incipient hematopoietic reconstitution after aplasia in patients with hematologic malignancies. J Cancer Res Clin Oncol. 2017;143(5):759-71. https://doi.org/10.1007/s00432017-2348-z.

14. Lin $H$, Lin HX, Ge N, Wang HZ, Sun R, Hu WH. Plasma uric acid and tumor volume are highly predictive of outcome in nasopharyngeal carcinoma patients receiving intensity modulated radiotherapy. Radiat Oncol. 2013;8(1): 121. https://doi.org/10.1186/1748-717X-8-121.

15. Du XJ, Chen L, Li WF, Tang LL, Mao YP, Guo R, et al. Use of pretreatment serum uric acid level to predict metastasis in locally advanced nasopharyngeal carcinoma. Head Neck. 2017;39(3):492-7. https://doi.org/1 0.1002/hed.24631

16. Amin MB. American joint committee on Cancer cancer staging manual. 8th ed. New York: Springer; 2016.

17. Lin S, Pan J, Han L, Guo Q, Hu C, Zong J, et al. Update report of nasopharyngeal carcinoma treated with reduced-volume intensitymodulated radiation therapy and hypothesis of the optimal margin. Radiother Oncol. 2014;110(3):385-9. https://doi.org/10.1016/j.radonc.2014. 01.011.

18. Eisenhauer EA, Therasse P, Bogaerts J, Schwartz LH, Sargent D, Ford R, et al. New response evaluation criteria in solid tumours: revised RECIST guideline (version 1.1). Eur J Cancer. 2009;45(2):228-47. https://doi.org/10.1016/j.ejca.2 008.10.026.

19. Ames BN, Cathcart R, Schwiers E, Hochstein P. Uric acid provides an antioxidant defense in humans against oxidant- and radical-caused aging and cancer: a hypothesis. Proc Natl Acad Sci U S A. 1981;78(11):6858-62. https://doi.org/10.1073/pnas.78.11.6858.

20. Gilbert LA, Hemann MT. DNA damage-mediated induction of a chemoresistant niche. Cell. 2010;143(3):355-66. https://doi.org/10.1016/j. cell.2010.09.043

21. Xu X, Rao GS, Groh V, Spies T, Gattuso P, Kaufman HL, et al. Major histocompatibility complex class I related chain a/B (MICA/B) expression in tumor tissue and serum of pancreatic cancer: role of uric acid accumulation in gemcitabine-induced MICA/B expression. BMC Cancer. 2011;11(1):194. https://doi.org/10.1186/1471-2407-11-194.
22. Vigano L, Darwish SS, Rimassa L, Cimino M, Carnaghi C, Donadon M, et al. Progression of colorectal liver metastases from the end of chemotherapy to resection: a new contraindication to surgery? Ann Surg Oncol. 2018;25(6): 1676-85. https://doi.org/10.1245/s10434-018-6387-8.

23. Li S, Wang Q, Mei J, Wang J, Zhong $X$, Ling $Y$, et al. Risk factors of extrahepatic progression after transarterial chemoembolization for hepatocellular carcinoma patients: a retrospective study in 654 cases. J Cancer. 2019;10(20): 5007-14. https://doi.org/10.7150/jca.35355.

24. Shiraishi T, Sasaki T, Ikeda K, Tsukada Y, Nishizawa Y, Ito M. Predicting prognosis according to preoperative chemotherapy response in patients with locally advanced lower rectal cancer. BMC Cancer. 2019;19(1):1222. https://doi.org/10.1186/s12885-019-6424-4.

25. Nougaret $S$, Rouanet $P$, Molinari N, Pierredon MA, Bibeau F, Azria D, et al. MR volumetric measurement of low rectal cancer helps predict tumor response and outcome after combined chemotherapy and radiation therapy. Radiology. 2012;263(2):409-18. https://doi.org/10.1148/ radiol.12111263.

26. Ma BB, Kam MK, Leung SF, Hui EP, King AD, Chan SL, et al. A phase II study of concurrent cetuximab-cisplatin and intensity-modulated radiotherapy in locoregionally advanced nasopharyngeal carcinoma. Ann Oncol. 2012;23(5): 1287-92. https://doi.org/10.1093/annonc/mdr401.

27. Farrell PJ. Epstein-Barr virus and Cancer. Annu Rev Pathol. 2019;14(1):29-53. https://doi.org/10.1146/annurev-pathmechdis-012418-013023.

28. Li J, Chen QY, He J, Li ZL, Tang XF, Chen SP, et al. Phase I trial of adoptively transferred tumor-infiltrating lymphocyte immunotherapy following concurrent chemoradiotherapy in patients with locoregionally advanced nasopharyngeal carcinoma. Oncoimmunology. 2015;4(2):e976507. https:// doi.org/10.4161/23723556.2014.976507

29. Ni W, Qi W, Xu F, Cao W, Xu C, Chen J, et al. Prognostic value of nasopharynx tumour volume in local-regional advanced nasopharyngeal carcinoma. Ann Transl Med. 2020;8(5):223. https://doi.org/10.21037/atm.2 020.01.03.

30. Chen BB, Lu SY, Peng H, Sun FF, Zhu J, Wang J, et al. Comparison of long-term outcomes and Sequelae between children and adult nasopharyngeal carcinoma treated with intensity modulated radiation therapy. Int J Radiat Oncol Biol Phys. 2020;106(4):848-56. https://doi. org/10.1016/j.jijrobp.2019.11.035.

\section{Publisher's Note}

Springer Nature remains neutral with regard to jurisdictional claims in published maps and institutional affiliations.

Ready to submit your research? Choose BMC and benefit from:

- fast, convenient online submission

- thorough peer review by experienced researchers in your field

- rapid publication on acceptance

- support for research data, including large and complex data types

- gold Open Access which fosters wider collaboration and increased citations

- maximum visibility for your research: over $100 \mathrm{M}$ website views per year

At $\mathrm{BMC}$, research is always in progress.

Learn more biomedcentral.com/submission 